gefährlichster Gegner für die Strategie der Kanzlerin), nicht vom „Super-Gau für die Demokratie“ (S. 37) reden: zum einen, weil ein „Gau“ bereits ein Superlativ ist, zum anderen, weil sie auf diese Weise die Grenzen zwischen Demokratie und Diktatur verwischt. Das gilt auch für „Dissidenten“ (S. 177). So heißen im Buch diejenigen, die Merkel Paroli bieten. Höhler behauptet, die Sprache verrate Merkel. Trifft das nicht (ebenso) auf sie zu? Die Macht der Sprache Höhlers ist der Sprache der Macht Merkels offenkundig unterlegen.

Die Frage, ob Angela Merkel in den Fußstapfen Helmut Kohls wandelt, wird nicht angemessen beantwortet. Höhler verweist auf - verhüllte - Kritik Kohls an der Kanzlerin. Aber ist sie mit ihrer Strategie und ihrer Konzeption - dem Machtwillen und der entschiedenen Europapolitik („Scheitert der Euro, dann scheitert Europa“) - nicht die beste „Kohlianerin“? Im Übrigen überschätzt Höhler den Einfluss Merkels massiv, wenn sie den Eindruck erweckt, die Politikerin könne Deutschland mehr oder weniger allein „umbauen“.

Das Werk, für den Tag geschrieben, belegt, wie eine eindrucksvolle Intellektuelle sich von ihren Emotionen mitreißen lässt. Prinzipieller Natur ist die Kritik an der „Allparteien-Koalition“ (S. 213), am „Allparteien-Konsens“ (S. 162) und am „Allparteien-Staat“ (S. 238). Diese Hinweise verdienen eine politikwissenschaftliche Reflexion. Treffen derartige Begriffe zu? Und wenn ja: Was sind die Gründe für eine solche Entwicklung? Liegt das in erster Linie an der Person von Angela Merkel?

Eckhard Jesse

\title{
Parteien nach Bundestagswahlen: verlässliche und kontinuierliche Analysen
}

Niedermayer, Oskar (Hrsg.): Die Parteien nach der Bundestagswahl 2009, Wiesbaden 2011, VS
Verlag für Sozialwissenschaften, 357 Seiten, € 39,95.

Zu den wichtigsten Publikationen im Bereich der Wahl- und Parteienforschung zählen sicherlich die Bände, die - unabhängig großer Schnittmengen - von den Arbeitskreisen „Wahlen und politische Einstellungen“ und „Parteienforschung“ der Deutschen Vereinigung für Politische Wissenschaft aus Anlass der Bundestagswahlen erarbeitet werden. Mit ihnen lassen sich die jeweiligen Bestimmungsgründe einer Wahl und das Abschneiden der Parteien kontinuierlich verfolgen. Nicht nur für die „Scientific Community“, sondern gerade auch für die wissenschaftlich orientierte Öffentlichkeit ist besonders die Kontinuität der Analysen von großem Vorteil. Seit 1993 sind allein 14 Bände im Rahmen des Arbeitskreises Parteienforschung erschienen. In denen, die den Bundestagswahlen gewidmet sind, finden sich regelmäßig Analysen zu den einzelnen Bundestagsparteien, der Entwicklung des Parteiensystems sowie eine Anzahl von variierenden Einzelaspekten. Nach dem schlechtesten Abschneiden der Volksparteien seit 1949 bei der Bundestagswahl 2009, ist es naheliegend, dass die Ursachen für diese Veränderungen das Schwerpunktthema des vorliegenden Buches bilden.

Der Sprecher des Arbeitskreises Parteienforschung und Herausgeber des Bandes, Oskar Niedermayer, ist einer der Garanten dieser Kontinuität, da er zuverlässig die Entwicklungen des Parteiensystems nach festen systematischen Kategorien vermisst. Trotz des Wandels zu einem „pluralistischen“ (S. 7) und „fluiden“ (S. 34) Fünf-Parteien-System schlussfolgert er keinesfalls - gegen einen weit verbreiteten Trend - dass das „Ende der Volksparteien“ ein- 
geläutet wäre“ (S. 16). Udo Zolleis und Josef Schmid halten es im Fall der CDU für zukunftsentscheidend, „ihre Kernanhängerschaft zu überzeugen“ (S. 53). Sie argumentieren, dass die Ursache für das schlechte Abschneiden der SPD darin liege, dass ihr die „Facharbeiterschaft den Rücken gekehrt“ (S. 53) habe. Dem widerspricht Manuela S. Kulick, die sich mit dem „Absturz der Volksparteien“ beschäftigt. Sie arbeitet heraus, dass sowohl bei der CDU als auch bei der SPD „in der Hauptsache aus ihrer Mitte“ (S. 217) Wähler verloren gegangen seien. Bei den Verlusten ehemaliger CDU- wie auch SPD-Wähler handle es sich zudem nicht um eine homogene Gruppe (vgl. S. 217). Sie betont, dass „die Gruppe der kurzfristig abgewanderten Wähler ein Potenzial“ aufweise, „welches von beiden Volksparteien auf vergleichsweise einfache Art wieder gewonnen werden kann“ (S. 218). Geradezu prophetische Präzision (auf dem Stand von Ende 2010) beweisen Ulrich von Alemann und Tim Spier, die die These vom Ende der Volkspartei SPD ebenfalls - sehr differenziert - zurückweisen, indem sie vor allem die Volatilität der Wähler betonen. „Schließlich könnte das Wahljahr 2010 in Hamburg und Baden-Württemberg eine Rückkehr der SPD in zwei Landesregierungen bescheren. Und die rot-grüne Minderheitsregierung im wichtigen Bundesland NRW stabilisiert sich und braucht Neuwahlen vorerst nicht zu fürchten" (S. 75) - wobei letzteres sich nur zur ersten Hälfte bewahrheitete.

$\mathrm{Zu}$ den Defiziten der Parteienforschung gehört seit längerem die Vernachlässigung der Parteien jenseits der parlamentarischen Repräsentation. Die kleinen Parteien bewegen sich regelmäßig außerhalb des Analyseinteresses der Parteienforschung. Auch in diesem Band wurden sie eher am Rande behandelt, indem Eckhard Jesse in seinem Beitrag kurz auf NPD und Piraten eingeht. Allerdings ist auch hier das Auswahlkriterium der Wahlerfolg, da diese Parteien die besten Ergebnisse der „sonstigen“ Parteien erreichten. Eine intensivere Beschäftigung mit diesen Parteien ist nicht nur aus phänomenologischer Sicht wünschenswert. Auch aus demokratietheoretischer Perspektive erscheint eine verstärkte Fokusbildung sinnvoll, da der Anteil der „sonstigen“ Parteien teilweise zweistellig ist und somit die Mehrheitsfindung und Koalitionsbildung in Parlamenten erheblich beeinflusst.

Wichtiger als die Analyse der Parteien ist die Auseinandersetzung um ihre Zulassung zu Wahlen, die Jesse eigentlich behandelt. Der Bundeswahlausschuss hat in einer Sitzung, die sich mit den Beschwerden zuvor nicht zugelassener Parteien zur Bundestagswahl befasste, vier Beschwerden nicht stattgegeben (insgesamt wurde 28-mal die Anerkennung als Partei verweigert). Dies waren die „Freie Union“, die „Grauen“, die „Partei“ und die „Bürgerpartei für alle“, wobei die Entscheidungen über die Freie Union, die Grauen und die Partei aus mehreren Gründen durchaus kritisch hinterfragt werden können ${ }^{1}$, auch wenn das Bundesverfassungsgericht in ständiger Rechtsprechung dem Verfahren nicht widerspricht. Hat der Bundeswahlausschuss früher ,eher großzügig“" über die Zulassung von Parteien zur Bundestagswahl entschieden und sich somit die Frage der Nichtteilnahme - ähnlich wie beim „negativen Stimmgewicht“ - in der Realität nicht gestellt, so ist die Virulenz nicht zu übersehen. Für nicht zugelassene Parteien besteht kein Rechtsschutz im Rahmen eines wie auch immer gearteten Eilverfahrens, ihre Zulassung nach einer Ablehnung durch den Bundeswahlausschuss zu erreichen und somit an einer Wahl teilzunehmen. ${ }^{2}$ „Dieser Sachverhalt ist

1 Vgl. Wilko Zicht, Zulassungsverfahren zur Bundestagswahl in der Kritik, 22. August 2009, http:// www.wahlrecht.de/news/2009/10.htm (Abruf am 7. Juli 2012).

2 Vgl. Florian Meinel, Chancengleichheit oder Kooptation? Der Zugang kleiner Parteien zur Bundestagswahl, in: ZParl, 41. Jg. (2010), H. 1, S. $67-76$. 
unhaltbar“ (S. 194), urteilt Eckhard Jesse. „Um für Unabhängigkeit zu sorgen, müsste eine unabhängige, etwa von Richtern besetzte Instanz eingeschaltet werden", lautet einer seiner Reformvorschläge (S. 194). Der Bundestag hat 2012 mit einem Gesetzentwurf zur Änderung des Grundgesetzes reagiert. ${ }^{3}$ Am 15. Juni 2012 hat der Bundesrat dem zugestimmt. ${ }^{4}$ Für Vereinigungen, die nicht als Parteien anerkannt wurden, wird eine Beschwerdemöglichkeit vor dem Bundesverfassungsgericht vorgesehen.

Viola Neu

3 Vgl. Deutscher Bundestag, Wahlbewerbern Rechtsweg nach Karlsruhe eröffnet, 24. Mai 2012, http://www.bundestag.de/dokumente/textarchiv/2012/39131399_kw21_de_rechtsschutz_wahlrecht/index.html (Abruf am 7. Juli 2012).

4 Vgl. Der Bundesrat, Bundesrat stimmt Grundgesetzänderung zu, 15. Juni 2012, http://www. bundesrat.de/cln_228/nn_1902780/DE/presse/pm/2012/088-2012.html?_nnn=true (Abruf am 7. Juli 2012).

\section{Parteien und ihre Frauenorganisationen: buntes Durcheinander mit etlichen Fehlern und handwerklichen Mängeln}

Kiefert, Cathleen: Politik ist eine viel zu ernste Sache, als dass man sie allein den Männern überlassen könnte. Die Frauenorganisationen in den deutschen Parteien (Nomos Universitätsschriften - Politik, Band 175), Nomos Verlagsgesellschaft, Baden-Baden 2011, 394 Seiten, $€ 64,-$.

Cathleen Kiefert hat für ihre umfangreiche Arbeit zur Organisation von Frauen in CDU, SPD, FDP, Linken und Grünen eine Fülle von Material zusammengetragen. Sie will nach „Zeiten der Frauenförderung und Gender Mainstreaming, Weltfrauenkonferenzen und einzelnen Frauen in höchsten politischen Ämtern der Welt“ die Frage beantworten: „Warum funktioniert das eigentlich immer noch nicht so richtig mit den Frauen und der Politik?" (S. 7). Dazu gliedert sie ihre Arbeit über die Arbeitsgemeinschaft Sozialdemokratischer Frauen (ASF), Frauen-Union (FU); Bundesfrauenrat und Bundesarbeitsgemeinschaft (BAG) Frauen, Liberale Frauen e.V. und Die Linke Sozialistische Arbeitsgemeinschaft (LISA) komparativ. Einer Einführung über Geschichte der Frauen und Frauenbewegung in Deutschland nach 1945 folgen in vier Großkapiteln Aussagen über Entstehung und Entwicklung, Aufgaben und Selbstverständnis, Aufbau und Organisation, Einfluss und Umsetzungsmöglichkeiten, aufgezeigt an Gleichstellung, Programmatik und Haltung zum Schwangerschaftsabbruch. Den Kapiteln stellt sie die jeweils einprägsamste Aussage als Motto voran. Schon mit der Wahl von griffigen, aber organisationsspezifischen Überschriften - „Das Frauenstatut ist Bestandteil der Satzung“ (Grüne) oder "Wer die menschliche Gesellschaft will, muss die männliche überwinden“ (Berliner Programm SPD 1989) und „Wer nicht kämpft, hat schon verloren (Süssmuth) “ - werden gravierende Unterschiede verdeckt. Der auf den ersten Blick ansprechende Aufbau überzeugt in der Ausführung nicht mehr. Die Gliederungspunkte werden weder eingeleitet noch zusammengefasst. Dies geschieht nur pauschal im Einleitungskapitel und zähflüssig im Ausblick. Die Chance, aus Zwischenergebnissen überleiten- 